\title{
Comparison of predictive scores of symptomatic intracerebral haemorrhage after stroke thrombolysis in a single centre
}

\author{
${ }^{1} \mathrm{~T}$. Watson-Fargie, ${ }^{2} \mathrm{D}$ Dai, ${ }^{3} \mathrm{MJ}$ MacLeod, ${ }^{4} \mathrm{JM}$ Reid \\ ${ }^{1}$ Senior Medical Student, University of Aberdeen, Aberdeen, UK; ${ }^{2}$ Research Scientist, Center for Paediatric Clinical Effectiveness and \\ PolicyLab, The Children's Hospital of Philadelphia, Philadelphia, USA; ${ }^{3}$ Senior Clinical Lecturer, University of Aberdeen, Aberdeen, UK; \\ ${ }^{4}$ Consultant Stroke Neurologist, Acute Stroke Unit, Aberdeen Royal Infirmary, Aberdeen, UK
}

\begin{abstract}
Symptomatic intracerebral haemorrhage following thrombolysis for ischaemic stroke causes significant morbidity and mortality. This study assessed which of four risk scores (SEDAN, HAT, GRASPS and SITS) best predicts symptomatic intracerebral haemorrhage.

Methods: Data from 431 patients treated at Aberdeen Royal Infirmary (20032013) were extracted from a thrombolysis database. Score performance was compared using area under the curve.

Results: Any intracerebral haemorrhage occurred in 12\% of patients (53/4/3); I I \% fulfilling the SITS-MOST symptomatic intracerebral haemorrhage definition (6/53), $34 \%$ the ECASS II definition (I8/53), and $43 \%$ the National Institute of Neurological Disorder and Stroke definition (23/53). Stroke severity, as defined by the National Institutes of Health Stroke Scale, significantly improved after 24 hours in patients without intracerebral haemorrhage, but not in those with. Significant symptomatic intracerebral haemorrhage predictors were age, glucose, stroke severity, hyperdense middle cerebral artery on CT scan, ASPECTS score and anti-platelet therapy. The haemorrhage after thrombolysis score performed best at predicting symptomatic intracerebral haemorrhage (area under the curve $0.67-0.78, \mathrm{p}<0.00 \mathrm{I})$.

Conclusion: The haemorrhage after thrombolysis score uses the least variables and has the best predictive value for symptomatic intracerebral haemorrhage. Using predictive scores for clinical decision making depends on estimation of overall benefits as well as risk.
\end{abstract}

KEYWORDS outcome prediction, predictive risk scores, stroke thrombolysis, symptomatic intracerebral haemorrhage

Correspondence to T Watson-Fargie Department of Neurosciences Aberdeen Royal Infirmary Aberdeen AB25 2ZN

UK

e-mail taylor.watson-fargie.10@ aberdeen.ac.uk

DECLARATION OF INTERESTS No conflict of interest declared.

\section{INTRODUCTION}

Stroke is the third most common cause of death in Scotland and accounts for approximately $5 \%$ of total UK NHS costs.' Thrombolytic therapy using intravenous alteplase is currently the only licensed therapy for acute ischaemic stroke (AIS). The most recent meta-analysis of thrombolysis for AIS demonstrates a $9.0 \%$ decrease in death and $4.2 \%$ decrease in dependency (defined as a modified Rankin Score of 3 to $6^{2}$ ) three months after stroke, when thrombolysis is given in the first three or six hours of symptom onset compared to control. ${ }^{3}$ However, the leading complication of thrombolysis is the development of symptomatic intracerebral haemorrhage $(\mathrm{SICH})$, which carries significant morbidity and mortality. Of those thrombolysed, 7.7\% developed $\mathrm{SICH}$, compared to $1.8 \%$ in control groups, with $\mathrm{SICH}$ accounting for most of the early deaths in the thrombolysed groups. ${ }^{3}$
This complication could be minimised if physicians could reliably predict which AIS patients might develop $\mathrm{SICH}$ after thrombolysis. Several studies have developed risk scores to try and estimate which patients are most at risk of $\mathrm{SICH}$ post-thrombolysis to inform thrombolysis decision making (e.g. the SEDAN, HAT, GRASPS and SITS scores $\left.^{4-7}\right)$. The aim of the current study was to test the performance of these scores and to see which was most valid in a population treated by a single stroke centre.

\section{METHODS}

\section{Study population}

Patients admitted to Aberdeen Royal Infirmary with a diagnosis of acute ischaemic stroke who were thrombolysed between 2003 and 2013 (majority enrolled from 2009 onwards) were entered in the Safe Implementation of Treatment in Stroke (SITS) database 
TWatson-Fargie, D Dai, MJ MacLeod et al.

$(n=43 \mathrm{I})$. This is a European-wide database for monitoring the safety of thrombolysis use in ischaemic stroke patients. ${ }^{8}$ Patients are entered as part of clinical audit and information collected includes age, gender, prestroke functional status (modified Rankin score), comorbidity, glucose, blood pressure and stroke severity (National Institutes of Health Stroke Scale - NIHSS ${ }^{9}$ ) at 0 hours, 2 hours, 24 hours and 7 days post-thrombolysis, plus findings from the initial and 24 hour postthrombolysis CT scan. The latter includes presence of intracerebral haemorrhage $(\mathrm{ICH})$ as previously described. 'This anonymised audit was approved by our local ethics committee.

\section{SICH risk prediction scores}

Four prediction scores were used in this study - SEDAN (blood Sugar, Early ischaemic changes, hyperDense artery sign, Age, and NIH Stroke Scale score), haemorrhage after thrombolysis (HAT), GRASPS (Glucose at presentation, Race [Asian], Age, Sex [male], systolic blood Pressure at presentation, and Severity of stroke at presentation [NIHSS] score) and SITS. Table I lists the various components of each risk score. ${ }^{4-7}$ With respect to the HAT score, it should be noted that to quantify the extent of early ischaemic change in the middle cerebral artery (MCA) territory, the Alberta Stroke Programme Early Computerised Tomography Score (ASPECTS) was utilised. This score evaluates ten anatomical sites within the MCA territory for signs of ischaemic change and produces a normal maximum score of 10 (no ischaemic changes), minus one point for each area with ischaemic changes..$^{1}$ In this study, it was assumed that an ASPECTS score of $\leq 7$ denoted MCA territory hypodensity of $>$ 1/3.

\section{SICH definitions}

$\mathrm{SICH}$ has numerous definitions. This study utilised only those definitions which are used by the respective $\mathrm{SICH}$ risk prediction scores. The SEDAN and HAT scores were developed using the European Cooperative Acute Stroke Study II (ECASS II) definition of $\mathrm{SICH}$, i.e. ICH with worsening in the NIHSS score of $\geq 4 .{ }^{5}$ The GRASPS score was developed using the National Institute of Neurological Disorder and Stroke (NINDS) definition: any ICH with a worsening in the NIHSS score of $\geq 1$." The SITS score uses the SITS-MOST definition, i.e. a local or remote parenchymal haemorrhage $(\mathrm{PH} 2$, a haemorrhage $>30 \%$ of the infarcted area with significant space-occupying effect) $)^{12}$ and worsening of NIHSS $\geq 4$."

\section{Predictor variables}

The following variables were extracted from the SITS database to evaluate significant univariate predictors of $\mathrm{SICH}$ and any $\mathrm{ICH}$ : age; gender; weight; systolic and diastolic blood pressures; baseline NIHSS score; glucose; cholesterol; prior previous stroke or transient ischaemic attack; history of hypertension; diabetes mellitus; chronic heart failure; atrial fibrillation; use of anti-hypertensive,

\begin{tabular}{|c|c|}
\hline $\begin{array}{l}\text { Risk score } \\
\text { (range of scores) }\end{array}$ & $\begin{array}{l}\text { Variables comprising each score } \\
\text { (points for score variable) }\end{array}$ \\
\hline $\begin{array}{l}\text { SEDAN } \\
(0-6)\end{array}$ & $\begin{array}{ll}\text { - } & \text { Admission blood glucose: } \geq \\
& 12 \mathrm{mmol} / \mathrm{L}(2) ; 8 . \mathrm{I}-\mathrm{I} 2 \mathrm{mmol} / \mathrm{L}(\mathrm{I}) \\
\text { - } & \text { Presence of early infarct sign on CT } \\
& \text { scan (I) } \\
\text { - } & \text { HDMCA on CT scan (I) } \\
\text { - } & \text { Age } \geq 75 \text { years (I) } \\
\text { - } & \text { NIHSS } \geq 10(\mathrm{I})\end{array}$ \\
\hline $\begin{array}{l}\text { HAT } \\
(0-5)\end{array}$ & $\begin{array}{l}\text { Diabetes mellitus or admission } \\
\text { blood glucose }>200 \mathrm{mg} / \mathrm{dL}(\mathrm{I}) \\
\text { - NIHSS: } \geq 20(2) ; 15-19(\mathrm{I}) \\
\text { - } \\
\text { Fraction of MCA territory } \\
\text { hypodensity: }>\mathrm{I} / 3(2) ; \mathrm{I} / 3(\mathrm{I})\end{array}$ \\
\hline $\begin{array}{l}\text { SITS } \\
(0-12)\end{array}$ & $\begin{array}{ll}\text { - } & \text { NIHSS: } \geq 13(2) ; 7-12(\mathrm{I}) \\
\text { - } & \text { Age } \geq 72 \text { years }(\mathrm{I}) \\
\text { - } & \text { Systolic Blood Pressure } \geq 146 \mathrm{mmHg} \\
& (\mathrm{I}) \\
\text { - } & \text { Weight } \geq 95 \text { kilograms }(\mathrm{I}) \\
\text { - } & \text { Blood glucose } \geq 10 \mathrm{mmol} / \mathrm{L}(2) \\
\text { - } & \text { Pre-stroke anti-coagulant therapy: } \\
& \text { aspirin and clopidogrel therapy (3); } \\
& \text { aspirin monotherapy (2) } \\
\text { - } & \text { Pre-stroke hypertension (I) } \\
\text { - } & \text { Onset-to-treatment time } \geq 180 \\
\text { minutes (I) }\end{array}$ \\
\hline $\begin{array}{l}\text { GRASPS } \\
(45-10 I)\end{array}$ & 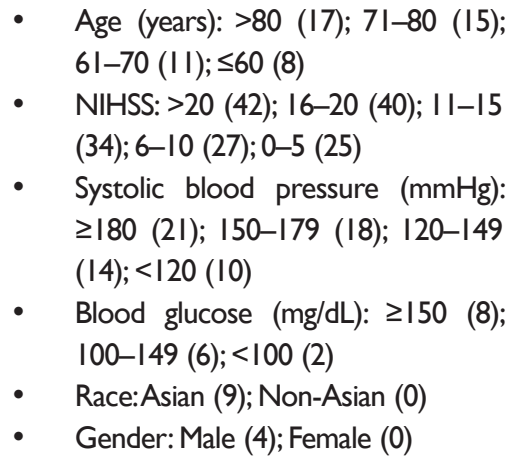 \\
\hline
\end{tabular}

HDMCA: hyperdense middle cerebral artery; CT: computerised tomography; NIHSS: National Institutes of Health Stroke Scale; MCA: middle cerebral artery.

anti-platelet or statin medication; symptom onset to treatment time, presence of a hyperdense middle cerebral artery (HDMCA) on CT scan and ASPECTS score.

\section{Statistical analysis}

Data are presented as either means or percentages unless otherwise stated. Comparisons between groups were made using the Chi-square test and t-test where appropriate with significance defined as $P<0.05$. 
TABLE 2 Univariate analysis to determine possible factors predisposing to any intracerebral haemorrhage and SICH following thrombolysis

\begin{tabular}{|c|c|c|c|c|}
\hline & $\begin{array}{c}\text { All } \\
(n=431)\end{array}$ & $\begin{array}{c}\text { Any haemorrhage } \\
(n=54)\end{array}$ & $\begin{array}{c}\text { NINDS SICH } \\
\text { Definition' }(n=23)\end{array}$ & $\begin{array}{c}\text { ECASS II SICH } \\
\text { Definition }^{2}(n=\mid 8)\end{array}$ \\
\hline Age (years) (SD) & $70.1(13.6)$ & $76.2^{m+\alpha}(10.4)$ & $77.9(9.3)$ & $77.2^{* *}(9.8)$ \\
\hline Male (\%) & $191(44)$ & $35(65)$ & $13(57)$ & II (6I) \\
\hline Weight (kg) (SD) & $76.3(16.6)$ & $76.9(18.3)$ & $73.6(17.3)$ & $71.5(17.1)$ \\
\hline $\begin{array}{l}\text { Systolic blood pressure } \\
(\mathrm{mmHg})(\mathrm{SD})\end{array}$ & $150.8(21.9)$ & I56.4 (I9.1) & $156.7(16.5)$ & $153.5^{*}(16.1)$ \\
\hline $\begin{array}{l}\text { Diastolic blood pressure } \\
(\mathrm{mmHg})(\mathrm{SD})\end{array}$ & $80.7(13.6)$ & $83.4(11.0)$ & $82.4(11.0)$ & $80.9(11.8)$ \\
\hline Baseline NIHSS ${ }^{3}$ (SD) & $11.2(6.7)$ & $14.0 *(6.0)$ & $16.5^{* *}(6.1)$ & $14.8^{* i * * *}(5.4)$ \\
\hline 24 hours NIHSS (SD) & $8.9(8.5)$ & $15 . I^{* * * *}(10.3)$ & $26.8^{* * 1 * x}(4.9)$ & $27.5^{\text {sike }}(5.3)$ \\
\hline $\begin{array}{l}\text { Onset to treatment } \\
\text { time }(\mathrm{min})(\mathrm{SD})\end{array}$ & $161.3(57.7)$ & $161(61.4)$ & I55.6 (7I.I) & I53.5 (7I.4) \\
\hline Glucose (mmol/L) (SD) & $6.8(2.2)$ & $7.5(2.5)$ & $7.7(2.9)$ & $7.9(3.3)$ \\
\hline $\begin{array}{l}\text { Cholesterol (mmol/L) } \\
\text { (SD) }\end{array}$ & $4.5(1.3)$ & $4.2(1.0)$ & $4.1(1.3)$ & $3.7(0.6)$ \\
\hline Previous stroke/TIA $(\%)$ & $72 / 367(20)$ & $16 / 49(33)^{*}$ & $6 / 23(26)$ & $5 / 18(28)$ \\
\hline Hypertension (\%) & $200 / 369(54)$ & 29/49 (59) & $13(57)$ & $10(56)$ \\
\hline Diabetes mellitus (\%) & $61 / 410(15)$ & $13 / 52(25)^{*}$ & $8(35)^{*+*}$ & $6(33)^{*}$ \\
\hline Chronic heart failure (\%) & $22 / 369(6)$ & $5 / 49(10)^{\text {sion }}$ & $2(9)$ & I (6) \\
\hline $\begin{array}{l}\text { Current non-smoking } \\
\text { status (\%) }\end{array}$ & $201 / 367(55)$ & $46 / 49(94)^{* * *}$ & $19(83)$ & $14(78)$ \\
\hline Atrial fibrillation (\%) & $84 / 370(24)$ & $17 / 49(35)^{*}$ & $6(26)$ & $5(28)$ \\
\hline $\begin{array}{l}\text { Anti-hypertensive } \\
\text { medication (\%) }\end{array}$ & $169 / 365(46)$ & $26 / 49(53)$ & $14(6 \mathrm{I})$ & II (6I) \\
\hline $\begin{array}{l}\text { Anti-platelet medication } \\
\text { (\%) }\end{array}$ & $132 / 348(38)$ & $30 / 48(63)^{\text {seste }}$ & $16(70)^{*}$ & $14(78)^{*+1 / x}$ \\
\hline Statin medication (\%) & $93 / 365(26)$ & I4/49 (29) & $6(26)$ & $5(28)$ \\
\hline $\operatorname{HDMCA}^{5}(\%)$ & II 4/430 (27) & $19 / 53(36)$ & $10(46)^{*}$ & $6(35)$ \\
\hline ASPECTS $^{6}$ (SD) & $9.3(1.2)$ & $8.6^{*}(1.5)$ & $7.7^{* * * *}(2.6)$ & $7.8^{* * * *}(2.5)$ \\
\hline
\end{tabular}

Means are expressed with standard deviations. Percentages are expressed with numerator and denominator. " $\mathrm{p}<0.05 ;$; $\mathrm{p}<0.01 ;$

'Any haemorrhage with worsening of NIHSS $\geq 1 ;{ }^{2}$ Any haemorrhage with worsening of NIHSS $\geq 4 ;{ }^{3}$ National Institutes of Health Stroke Scale; ${ }^{4}$ Transient ischaemic attack; ${ }^{5}$ Hypodense middle cerebral artery; ${ }^{6}$ Alberta Stroke Programme Early Computerised Tomography Score

Univariate analysis was performed to identify potential variables associated with $\mathrm{SICH}$ in our population. Multivariate analysis was not performed in view of the sample size, and because we wanted to test previously established risk scores rather than develop any new predictive models or scores. We conducted a posthoc bootstrapping analysis of 1000 iterations to compare the predictive performances of the four $\mathrm{SICH}$ risk scores using the area under the receiveroperator curve (AUROC). ${ }^{13}$ An AUROC $=0.5$ indicates the risk score performs no better than chance, whereas an AUROC of I indicates perfect discrimination. The best risk score was defined as the score with the largest statistically significant AUROC using analyses of variance. ${ }^{14}$ Analyses were conducted using SAS software, version 9.3 (SAS Institute, Inc., Cary, NC, USA).

\section{RESULTS}

Data on a total of 43I acute ischaemic stroke patients who received intravenous alteplase and were entered into the SITS database were analysed in this study. From comparison SITS data the baseline stroke severity and characteristics are similar to other patients receiving thrombolysis in the UK (mean age 70 vs 70 years, $78 \%$ free of disability pre-stroke [modified Rankin score <2] vs $86 \%$, NIHSS I 0 vs 12 and $40 \%$ total anterior circulation syndrome vs $46 \%$ respectively). Any ICH occurred in I2\% (53/4I3) of patients; of these only II\% (6/53) fulfilled the SITS-MOST definition of SICH," $34 \%$ (I8/53) the ECASS II definition, ${ }^{4}$ and $43 \%$ (23/53) the NINDS definition. ${ }^{10}$ Mean NIHSS significantly improved in patients without ICH after 24 hours (I0.8 [SD 6.6] to 8.0 [SD 7.8], $p<0.0001$ ), whereas there was a non- 
significantly increased NIHSS in patients who had any ICH (I7.0 [SD I0.8] vs I4.0 [SD 6.I], p=0.08). Univariate analysis identified several significant predictors of $\mathrm{SICH}$ as defined by NINDS and ECASS II definitions following thrombolysis (Table 2); age, glucose, NIHSS, diabetes mellitus, HDMCA on CT scan, ASPECTS score and antiplatelet therapy prior to stroke onset.

Only six patients fulfilled the SITS-MOST definition of $\mathrm{ICH}$ (clinical data not shown in view of lack of statistical power). Additional univariate predictors for developing any ICH include previous cerebral ischaemic event, nonsmoking status, congestive cardiac failure and atrial fibrillation. Onset to treatment time, gender, glucose levels and anti-hypertensive medication were not significant predictors.

The median values for the following scores are: SEDAN 2 (inter-quartile ratio [IQR] I-2); HAT I (IQR 0-2); GRASPS 69 (IQR 62-75) and SITS 4 (IQR 3-6). The HAT score was the best predictor score regardless of definition of SICH (Table 3). The SITS score was the next best for an ECASS II definition of $\mathrm{SICH}$ whereas the GRASP score was the next best for the NINDS definition.

\section{DISCUSSION}

This study aimed to compare the accuracy of four different scoring systems to predict $\mathrm{SICH}$ in patients admitted to our hospital following thrombolysis. The HAT score is the simplest and best performing risk score regardless of $\mathrm{SICH}$ definition. This suggests that the variables used in this score (i.e. diabetes mellitus or glucose level, stroke severity and extent of early ischaemic change) are the strongest independent predictors of $\mathrm{SICH}$. Higher NIHSS (i.e. stroke severity) likely correlates with the volume of ischaemic tissue at risk for haemorrhagic transformation. ${ }^{15}$ The degree of visible hypodensity on the pre-treatment CT is thought to represent cytotoxic oedema and possible irreversible injury, which is associated with ICH after thrombolysis. ${ }^{16}$ Experimental studies showed that diabetes mellitus and hyperglycaemia are associated with blood-brain barrier and microvascular impairments, as well as increased haemorrhagic infarct conversion after reperfusion. ${ }^{17} \mathrm{~A}$ recent meta-analysis suggests that admission glucose but no prior diabetes predicts post-thrombolysis $\mathrm{SICH} .^{18}$ However, admission glucose may be a surrogate marker of brain infarction severity (as a stress response) rather than a causal factor.

Our population variables, such as onset to treatment time, age, blood pressure or hypertension and gender add little predictive power. This is despite some of these variables being univariate predictors of any $\mathrm{ICH}$ in our population.Variables found to be significant predictors in univariate analysis may be associated with $\mathrm{SICH}$, but may
TABLE 3 Area under the receiver-operator curve values for the definitions of symptomatic intracerebral haemorrhage. Data expressed as mean $(95 \% \mathrm{Cl})$.

\begin{tabular}{|l|c|c|c|}
\hline $\begin{array}{l}\text { SICH risk } \\
\text { prediction } \\
\text { scores }\end{array}$ & \multicolumn{3}{|c|}{ AUROC of SICH Definitions } \\
\hline & $\begin{array}{c}\text { SITS-MOST } \\
(\mathrm{n}=6)\end{array}$ & $\begin{array}{c}\text { ECASS II } \\
(\mathrm{n}=8)\end{array}$ & $\begin{array}{c}\text { NINDS } \\
(\mathrm{n}=23)\end{array}$ \\
\hline SEDAN & 0.62 & 0.67 & 0.72 \\
& $(0.6 \mathrm{I}-0.63)$ & $(0.65-0.69)$ & $(0.7 \mathrm{I}-0.73)$ \\
\hline HAT & 0.67 & 0.73 & 0.78 \\
& $(0.65-0.69)$ & $(0.7 \mathrm{I}-0.75)$ & $(0.75-0.8 \mathrm{I})$ \\
\hline GRASPS & 0.65 & 0.69 & 0.74 \\
& $(0.63-0.67)$ & $(0.66-0.70)$ & $(0.72-0.76)$ \\
\hline SITS & 0.68 & 0.72 & 0.72 \\
& $(0.66-0.69)$ & $(0.70-0.74)$ & $(0.70-0.73)$ \\
\hline
\end{tabular}

not be causally related. Multivariate analysis, as used in development of SICH predictor scores (e.g. HAT or SEDAN), identifies independent predictor variables. Many of these predictors (e.g. age, gender, non-smoking status and chronic heart failure) have been found in previous studies to be risk factors for $\mathrm{ICH}$, although not necessarily SICH post-thrombolysis." In particular, although blood pressure is a variable in the GRASPS and SITS scores, a recent meta-analysis of studies of $\mathrm{SICH}$ could not identify blood pressure even as a significant univariate predictor. $^{19}$ Onset to treatment time is a significant factor in estimating the benefit of thrombolysis with an approximate halving of benefit every 90 minutes. The risk of SICH appears mostly constant in the 0-6 hour time window, which may be one reason the SITS score performs less well than the HAT score. ${ }^{3,19,20}$ The majority of patients (96\%) in this series were treated with intravenous thrombolysis within the current accepted time window of within 4.5 hours of symptom onset. Systems should be optimised to maximise early delivery of stroke thrombolysis to maximise benefit. We did not find any difference in ASPECTS score with regard to onset to treatment time (data not shown), but the degree of appearance of early ischaemic change should be time-dependent, although other factors such as collateral circulation may affect ASPECTS score.

A meta-analysis by Whiteley et al. ${ }^{19}$ showed that factors which may predict $\mathrm{SICH}$ following thrombolysis are those which also predict poor outcome following a stroke. The authors further concluded that predicting which patients will develop SICH may be difficult. Those patients predicted to have a high risk of $\mathrm{SICH}$ may not have a poor outcome following thrombolysis, and so clinical decision making is difficult. ${ }^{19}$ A further recent analysis of patients from the third International Stroke Trial (IST-3) trial demonstrates that patients predicted to be at highest risk of developing $\mathrm{SICH}$ still achieve a net population benefit from receiving thrombolysis. ${ }^{21}$ Similar 
results to those found in this paper have been demonstrated by Strbian et al., ${ }^{22}$ who showed that the SEDAN scorehad the highest predictive power in 3012 patients with a $7.3 \%$ rate of $\mathrm{SICH}$. They also concluded that none of the predictive scores they assessed, including HAT, SEDAN, GRASPS and SITS, had better than moderate performance when analysed using the area under the curve, similar to the findings from IST-3. ${ }^{2}$

This current study had some limitations. Patients admitted to a single stroke unit were studied and it is uncertain whether this study population is generalisable. Despite this the demographics and stroke severity of patients treated at our centre were similar to the average UK patient. Furthermore, this study may be underpowered for some of the analyses. The differing $\mathrm{SICH}$ definitions are a further limitation as this prevents precise comparison between $\mathrm{SICH}$ prediction scores. There is debate as to which SICH definition is the most relevant. The SITS-MOST definition seems a very narrow definition, being present in only $1.4 \%$ of the patients in this study, whereas the NINDS definition is too liberal as NIHSS can worsen by I point purely due to oedema after acute ischaemic stroke. The ECASS II definition is recognised as being the most pertinent in predicting poor and fatal outcomes. ${ }^{23}$ Furthermore it is clear that minor haemorrhages (e.g. PHI), which can be excluded from some $\mathrm{SICH}$ definitions, may be associated with worse outcomes, and survivors of $\mathrm{SICH}$ tend to have poorer outcomes. ${ }^{23}$

In conclusion, this study demonstrates that age, glucose, stroke severity (as defined by the NIHSS score), diabetes mellitus, HDMCA on CT scan, ASPECTS and prior antiplatelet therapy are univariate predictors of developing $\mathrm{SICH}$. The HAT score uses the simplest variables and appears to have the best predictive value for $\mathrm{SICH}$ in this population, regardless of definition. Despite this the HAT score does not assess overall benefit of thrombolytic therapy in addition to risk of harm. In particular it is notable that the increase in mortality at 7 days after thrombolysis, mostly due to $\mathrm{SICH}$, appears to be negated by improved survival at six months in both the IST-3 trial ${ }^{24}$ and the most recent systematic review of alteplase for ischaemic stroke. Further studies are required to determine whether $\mathrm{SICH}$ prediction scores should influence clinical decision-making in regard to the use of thrombolytic therapy for AIS. At a minimum, the HAT score could be used to estimate the risk of $\mathrm{SICH}$ in a given patient when discussing the risk and benefit of thrombolysis with stroke patients or their relatives.

\section{REFERENCES}

I Saka O, McGuire A, Wolfe C. Cost of stroke in the United Kingdom. Age and Ageing 2009; 38: 27-32. http://dx.doi.org//0.1093/ageing/afn28I

2 Van Swieten JC, Koudstaal PJ, Visser MC et al. Interobserver agreement for the assessment of handicap in stroke patients. Stroke 1988; 19: 604-7.

3 Wardlaw JM, Murray V, Berge E et al. Recombinant tissue plasminogen activator for acute ischaemic stroke: an updated systematic review and meta-analysis. Lancet 20I2 23; 379: 2364-72. http://dx.doi. org/I0.I0I6/S0I40-6736(I2)60738-7

4 Strbian D, Engelter S, Michel $P$ et al. Symptomatic intracranial hemorrhage after stroke thrombolysis: The SEDAN score. Ann Neurol 2012; 7I: 634-4I. http://dx.doi.org/I0.1002/ana.23546

5 Lou M, Safdar A, Mehdiratta $M$ et al. The HAT score a simple grading scale for predicting hemorrhage after thrombolysis. Neurology 2008; 7I: 14I7-23. http://dx.doi.org//0.12I2/0I. wnl.0000330297.58334.dd

6 Menon BK, Saver JL, Prabhakaran S et al. Risk score for intracranial hemorrhage in patients with acute ischemic stroke treated with intravenous tissue-type plasminogen activator. Stroke 2012; 43: 2293-9. http://dx.doi.org/ I0. I I 6 I/STROKEAHA. I I 2.6604I5

7 Mazya M, Egido JA, Ford GA et al. Predicting the risk of symptomatic intracerebral hemorrhage in ischemic stroke treated with intravenous alteplase: Safe Implementation of Treatments in Stroke (SITS) symptomatic intracerebral hemorrhage risk score. Stroke 20I2;43:I524-3 I http://dx.doi.org/I 0. I I 6 I/STROKEAHA. I I I.6448I5

8 SITS. Safe Implementation of Treatments in Stroke. http://www. sitsinternational.org (accessed 30/7/20I3).

9 Brott T,Adams HP, Olinger CP et al. Measurements of acute cerebral infarction: a clinical examination scale. Stroke 1989; 20: 864-70.
10 Barber PA, Demchuk AM, Zhang J et al. Validity and reliability of a quantitative computed tomography Score in predicting outcome of hyperacute stroke before thrombolytic therapy. Lancet 2000; 355 : 1670-4.

II McKinney JS, Cucchiara B. Risk scores for predicting postthrombolysis intracerebral hemorrhage. US Neurology 20I0; 5: 39-40.

12 Trouillas, P, von Kummer, R. Classification and pathogenesis of cerebral hemorrhages after thrombolysis in ischemic stroke. Stroke 2006; 37: 556-6I.

I3 Hanley JA, McNeil BJ. The meaning and use of the area under a receiver operating characteristic (ROC) curve. Radiology 1982; 143: 29-36.

14 DeLong ER, DeLong DM, Clark-Pearson DL. Comparing the area under two or more correlated receiver operating characteristic curves: a nonparametric approach. Biometrics 1988; 44: 837-45.

15 The NINDS t-PA Stroke Study Group. Intracerebral hemorrhage after intravenous t-PA therapy for ischemic stroke. Stroke 1997; 28: 2109-18.

16 Grond M,von Kummer R, Sobesky J et al. Early x-ray hypoattenuation of brain parenchyma indicates extended critical hypoperfusion in acute stroke. Stroke 2000; 31: 133-9.

17 Hawkins BT, Lundeen TF, Norwood KM et al. Increased bloodbrain barrier permeability and altered tight junctions in experimental diabetes in the rat: contribution of hyperglycaemia and matrix metalloproteinases. Diabetologia 2007; 50: 202-II.

18 Desilles JP, Meseguer E, Labreuche J et al. Diabetes mellitus, admission glucose, and outcomes after stroke thrombolysis: a registry and systematic review. Stroke 2013; 44: 1915-23. http:// dx.doi.org/ I0.1 I6I/STROKEAHA. I I I.0008/3 
TWatson-Fargie, D Dai, MJ MacLeod et al.

19 Whiteley WN, Slot KB, Fernandes $P$ et al. Risk factors for intracranial hemorrhage in acute ischemic stroke patients treated with recombinant tissue plasminogen activator: a systematic review and meta-analysis of 55 studies. Stroke 20I2; 43: 2904-9. http:// dx.doi.org/ I0. I I6I/STROKEAHA. I I2.66533 I

20 Lees KR, Bluhmki E, von Kummer R et al. Time to treatment with intravenous alteplase and outcome in stroke: an updated pooled analysis of ECASS, ATLANTIS, NINDS, and EPITHET trials. Lancet 2010 15; 375: 1695-703. http://dx.doi.org//0.1016/SOI406736(I0)6049I-6

21 Whiteley WN, Thompson D, Murray G et al. Targeting recombinant tissue-type plasminogen activator in acute ischemic stroke based on risk of intracranial hemorrhage or poor functional outcome: an analysis of the Third International Stroke Trial. Stroke 20I4; 45: I0006. http://dx.doi.org/ I 0.I I6I/STROKEAHA. I I 3.004362
22 Strbian D, Michel P, Seiffge DJ et al. Symptomatic intracranial hemorrhage after stroke thrombolysis: comparison of prediction scores. Stroke 20I4; 45: 752-8. http://dx.doi.org//0.II6I/ STROKEAHA.II3.003806

23 Strbian D, Sairanen T, Meretoja A et al. Patient outcomes from symptomatic intracerebral hemorrhage after stroke thrombolysis. Neurology. 201I; 77: 34I-8. http://dx.doi.org//0.1212/ WNL.0b0I3e3182267b8c

24 IST-3 Collaborative Group, Sandercock P, Wardlaw JM et al. The benefits and harms of intravenous thrombolysis with recombinant tissue plasminogen activator within $6 \mathrm{~h}$ of acute ischaemic stroke (the Third International Stroke Trial [IST-3]): a randomised controlled trial. Lancet 20I2; 379: 2352-63. http://dx.doi.org/I0.10I6/SOI406736(I2)60768-5 